\title{
Arithmetic and ergodic properties of 'flipped' continued fraction algorithms
}

\author{
by \\ K. Dajani (Utrecht), D. Hensley (College Station, TX), \\ C. Kraaikamp (Delft) and V. Masarotto (Padova and Delft)
}

1. Introduction. It is well-known that every real number $x$ can be written as a finite (in case $x \in \mathbb{Q}$ ) or infinite (regular) continued fraction expansion ( $\mathrm{RCF}$ ) of the form

$$
x=a_{0}+\frac{1}{a_{1}+\frac{1}{a_{2}+\cdots+\frac{1}{a_{n}+\cdots}}},
$$

where $a_{0} \in \mathbb{Z}$ is such that $x-a_{0} \in[0,1)$, i.e. $a_{0}=\lfloor x\rfloor$, and $a_{n} \in \mathbb{N}$ for $n \geq 1$. The expansion (1), abbreviated by $x=\left[0 ; a_{1}, a_{2}, \ldots, a_{n}, \ldots\right]$, is infinite if and only if $x \in \mathbb{R} \backslash \mathbb{Q}$; in case $x \in \mathbb{Q}$ - essentially due to Euclid's algorithm $-x$ has two finite expansions.

The partial quotients $a_{n}$ are given by

$$
a_{n}=a_{n}(x)=\left\lfloor 1 / T^{n-1}(x)\right\rfloor \quad \text { if } T^{n-1}(x) \neq 0,
$$

where $T:[0,1) \rightarrow[0,1)$ is the continued fraction (or Gauss) map, defined by

$$
T(x)=1 / x-\lfloor 1 / x\rfloor \quad \text { if } x \neq 0,
$$

and $T(0)=0$; for more information, proofs, etc. on continued fractions; see also the monographs [DK02, H06, IK02, K56, P50, RS92.

Apart from the regular continued fraction expansion, there are (uncountably) many other continued fraction algorithms. Examples of these are Nakada's $\alpha$-expansions, the backward continued fraction, the continued fraction expansion with odd partial quotients (and the continued fraction with even partial quotients), Minkowski's diagonal expansion, etc. In this

2010 Mathematics Subject Classification: Primary 28D05, 11K50.

Key words and phrases: continued fractions, ergodicity, $\sigma$-finite infinite invariant measures. 
paper we will introduce and study a new class of continued fraction expansions: the D-expansions, or flipped expansions. Although the definition will be very simple, we will see that this class of expansions will contain a large class of different continued fraction algorithms, such as the (folded) $\alpha$-expansions, a variation on the backward continued fraction, the odd and even continued fraction expansions. However, Minkowski's diagonal expansion [Pi55] and the Bosma-Selenius optimal continued fraction expansion [B87] are not $D$-expansions.

1.1. Definition of $D$-expansions. Let $D \subset[0,1]$ be a Borel measurable subset of the unit interval. We define the map $T_{D}:[0,1) \rightarrow[0,1)$ by

$$
T_{D}(x):= \begin{cases}\lfloor 1 / x\rfloor+1-1 / x & \text { if } x \in D, \\ 1 / x-\lfloor 1 / x\rfloor & \text { if } x \in[0,1) \backslash D .\end{cases}
$$

Set

$$
\begin{aligned}
& \varepsilon_{1}=\varepsilon_{1}(x)= \begin{cases}-1 & \text { if } x \in D, \\
+1 & \text { if } x \in[0,1) \backslash D,\end{cases} \\
& d_{1}=d_{1}(x)= \begin{cases}\lfloor 1 / x\rfloor+1 & \text { if } x \in D, \\
\lfloor 1 / x\rfloor & \text { if } x \in[0,1) \backslash D .\end{cases}
\end{aligned}
$$

It follows from (4) that

$$
T_{D}(x)=\varepsilon_{1}\left(1 / x-d_{1}\right) .
$$

Now setting, for $n \geq 1$ for which $T_{D}^{n-1}(x) \neq 0$,

$$
d_{n}=d_{1}\left(T_{D}^{n-1}(x)\right), \quad \varepsilon_{n}=\varepsilon_{1}\left(T_{D}^{n-1}(x)\right),
$$

we find that

$$
x=\frac{1}{d_{1}+\varepsilon_{1} T_{D}(x)}=\frac{1}{d_{1}+\frac{\varepsilon_{1}}{d_{2}+\cdots+\frac{\varepsilon_{n-1}}{d_{n}+\varepsilon_{n} T_{D}^{n}(x)}}} .
$$

We denote the continued fraction expansion of $x$ thus obtained by

$$
x=\left[0 ; 1 / d_{1}, \varepsilon_{1} / d_{2}, \varepsilon_{2} / d_{3}, \ldots\right] .
$$

The proof that the convergents of this continued fraction expansion of $x$ (obtained by setting $T_{D}^{n}(x)$ equal to 0 in (5)) indeed converge to $x$, as indicated by (6), is postponed to Section 2. In that section other classical basic properties of continued fractions will be derived for $D$-expansions.

\subsection{Examples of $D$-expansions}

1.2.1. Nakada's $\alpha$-continued fractions. In 1981, H. Nakada [N81] generalized the RCF-expansion to a new class of continued fraction expansions, 
which is now well known as Nakada's $\alpha$-expansions. The underlying continued fraction transformation $T_{\alpha}$, for $\alpha \in[0,1]$, is given by

$$
T_{\alpha}(x)=|1 / x|-\lfloor|1 / x|+1-\alpha\rfloor \quad \text { for } \alpha-1<x<\alpha, x \neq 0,
$$

and $T_{\alpha}(0)=0$.

In 1997, Marmi, Moussa and Yoccoz [MMY97] modified Nakada's $\alpha$ expansions to the folded or Japanese continued fractions, with underlying map

$$
\widetilde{T}_{\alpha}(x)=\left|1 / x-\lfloor 1 / x\rfloor_{\alpha}\right| \quad \text { for } \alpha-1<x<\alpha, x \neq 0 ; \quad \widetilde{T}_{\alpha}(0)=0,
$$

where $\lfloor x\rfloor_{\alpha}=\min \{p \in \mathbb{Z}: x<\alpha+p\}$. We have $\widetilde{T}_{\alpha}=\left|T_{\alpha}\right|$. Clearly, folded $\alpha$-expansions can also be described as $D$-expansions with

$$
D=\bigcup_{n=1}^{\infty}\left(\frac{1}{n+1}, \frac{1}{n+\alpha}\right]
$$

see also Figure 1 .

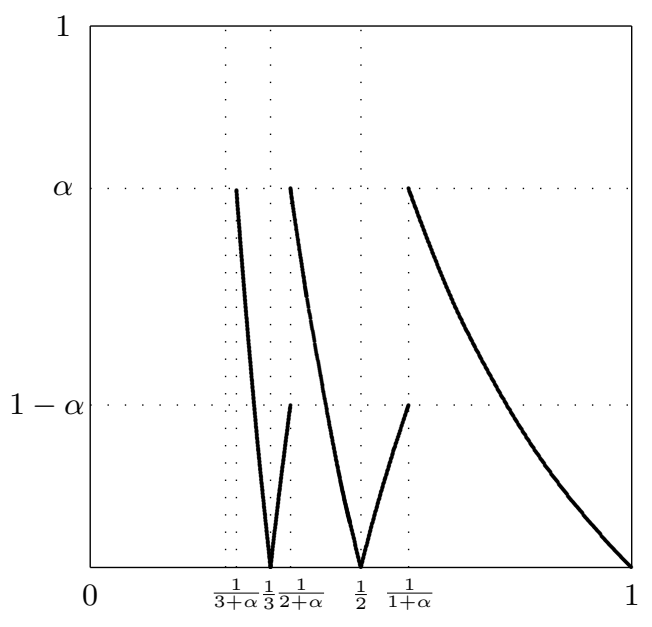

Fig. 1. The 'folded' $\alpha$-continued fraction map $\widetilde{T}_{\alpha}$

Note that for folded $\alpha$-expansions the interval $[0, \max \{\alpha, 1-\alpha\}]$ is an attractor, i.e., $\widetilde{T}_{\alpha}([0, \max \{\alpha, 1-\alpha\}])=[0, \max \{\alpha, 1-\alpha\}]$. Furthermore, if $x \notin[0, \max \{\alpha, 1-\alpha\}]$, then $\widetilde{T}_{\alpha}(x) \in[0, \max \{\alpha, 1-\alpha\}]$. So it is natural to restrict one's attention to the interval $[0, \max \{\alpha, 1-\alpha\}]$.

1.2.2. Backward continued fractions. Let $D=[0,1)$ and $x \in[0,1)$. In this case $[0,1) \backslash D=\emptyset$, so we always use the map

$$
T_{D}(x)=1+\lfloor 1 / x\rfloor-1 / x,
$$


and we will get an expansion of the form

$$
x=\frac{1}{d_{1}+\frac{-1}{d_{2}+\ldots}}=\left[0 ; 1 / d_{1},-1 / d_{2}, \ldots\right] ;
$$

see also Figure 2 It is a classical result that every $x \in[0,1) \backslash \mathbb{Q}$ has a unique backward continued fraction expansion

$$
x=1-\frac{1}{c_{1}-\frac{1}{c_{2}-\ldots}}=\left[0 ;-1 / c_{1},-1 / c_{2}, \ldots\right],
$$

where the $c_{i}$ s are all integers greater than 1; see AF84. This continued fraction is generated by the map

$$
T_{b}(x)=\frac{1}{1-x}-\left\lfloor\frac{1}{1-x}\right\rfloor,
$$

which we obtain from $T_{D}$ via the isomorphism $\psi: x \mapsto 1-x$, i.e., $\psi \circ T_{b}=$ $T_{D} \circ \psi ;$ see also Figure 2 .
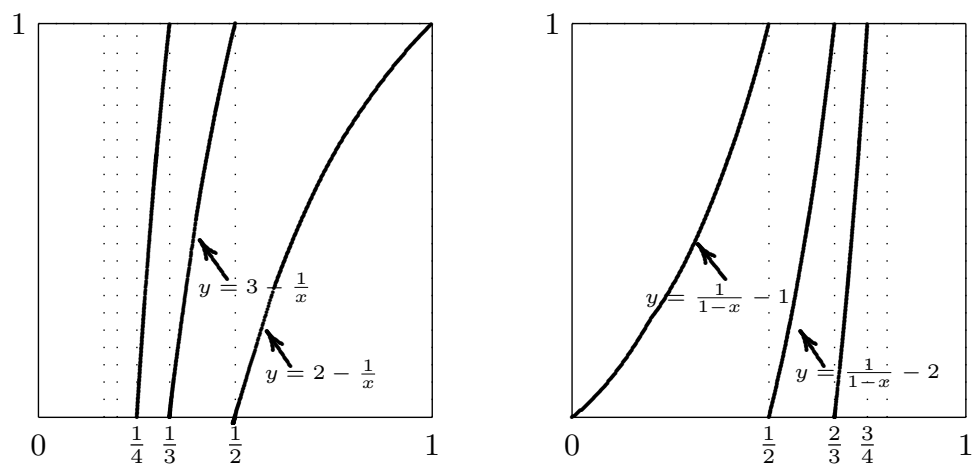

Fig. 2. The $D$-backward continued fraction map $T_{D}$ (left), and the classical backward continued fraction map $T_{b}$ (right)

1.2.3. Odd and even continued fractions. Setting

$$
D:=D_{\text {odd }}=\bigcup_{n \text { even }}\left[\frac{1}{n+1}, \frac{1}{n}\right),
$$

one easily finds that the $D$-expansion for every $x \in[0,1)$ only has odd partial quotients $d_{n}$. In case $D:=D_{\text {even }}=D_{\text {odd }}^{c}$, the partial quotients are always even. The maps $T_{D_{\text {odd }}}$ resp. $T_{D_{\text {even }}}$ have been studied by Fritz Schweiger S82, S84].

1.2.4. Continued fraction expansion without a particular digit. Using the definition of $D$-expansions, it is very easy to find continued fraction 
expansions in which a particular digit is always missing in the expansion of any $x \in[0,1)$. Indeed, fix a positive integer $\ell$, and suppose that we want an expansion in which the digit $\ell$ never appears, that is, $a_{n} \neq \ell$ for all $n \geq 1$. Now just take $D=(1 /(l+1), 1 / l]$ to get such an expansion. Figure 3 represents the map which yields the expansion without digit 2 .

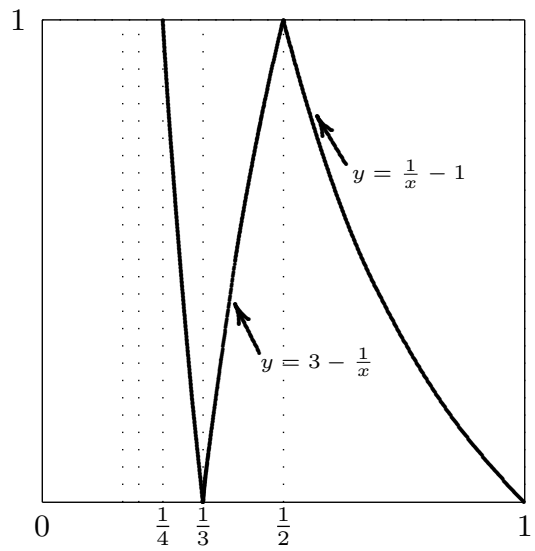

Fig. 3. The $D$-expansion map $T_{D}$ corresponding to $D=(1 / 3,1 / 2]$

1.3. Outline of the paper. In the next section we study the basic properties of $D$-expansions. In particular, we will see that two 'operations' on partial quotients (singularizations and insertions) can be used to understand the relation between the $D$-expansion (for a particular set $D$ ) and the RCF-expansion of any $x \in[0,1)$. Among other things we obtain the result (classical for RCF) that $x$ has an eventually periodic $D$-expansion if and only if $x$ is an irrational root of a quadratic equation.

In Section 3 we study the metric properties of $D$-expansions. Due to results by Rényi, Rychlik, Thaler, and Zweimüller, for each measurable set $D \subset[0,1]$ there exists a measure $\mu_{D}$ on $[0,1)$ which is $T_{D}$-invariant, and for which the dynamical system $\left([0,1), \mu_{D}, T_{D}\right)$ is ergodic. In case $D$ contains a neighborhood of 1 which is a subset of the support of $\mu_{D}$, the measure $\mu_{D}$ is $\sigma$-finite infinite, while it is a finite measure otherwise. In the final section we will see that for many choices of $D$ it is still an open question to determine the density $d_{D}$ of the measure $\mu_{D}$. For example, the explicit density of Nakada's $\alpha$-expansion is still unknown when $\alpha \in(0, \sqrt{2}-1]$; see [LM08, NN08. Using an approach of Geon Choe ([C00, C05]) we will see that for some 'easy' choices of $D$ the density $d_{D}$ seems to have a surprising shape.

2. Basic properties of $D$-expansions. Singularizations and insertions are two algebraic procedures that work via manipulation of partial quotients. 
They are both classical (cf. [P50]), and have been used a lot lately; see for example [K91, DK00, HK02, S04. We will use them to built a link between $D$-expansions and regular continued fractions, from which the elementary properties of $D$-expansions easily follow.

\subsection{Singularizations and insertions}

2.1.1. Singularizations. Let $a, b$ be positive integers, $\varepsilon= \pm 1$, and let $\xi \in[0,1)$. A singularization is based on the identity

$$
a+\frac{\varepsilon}{1+\frac{1}{b+\xi}}=a+\varepsilon+\frac{-\varepsilon}{b+1+\xi} .
$$

To see the effect of a singularization on a continued fraction expansion, let $x \in[0,1)$ have continued fraction expansion

$$
x=\left[a_{0} ; \varepsilon_{0} / a_{1}, \varepsilon_{1} / a_{2}, \varepsilon_{2} / a_{3}, \ldots\right],
$$

and suppose that for some $n \geq 0$ one has

$$
a_{n+1}=1, \quad \varepsilon_{n+1}=+1, \quad a_{n}+\varepsilon_{n} \neq 0,
$$

i.e.,

(9) $\left[0 ; \varepsilon_{0} / a_{1}, \ldots, a_{n}, \ldots\right]=\left[a_{0} ; \varepsilon_{0} / a_{1}, \ldots, \varepsilon_{n-1} / a_{n}, \varepsilon_{n} / 1,1 / a_{n+2}, \ldots\right]$.

Singularization then changes the continued fraction expansion in (8) into

$$
\left[a_{0} ; \varepsilon_{0} / a_{1}, \ldots, \varepsilon_{n-1} /\left(a_{n}+\varepsilon_{n}\right),-\varepsilon_{n} /\left(a_{n+2}+1\right), \ldots\right] .
$$

As in the case of the regular continued fraction, finite truncation yields the sequence of convergents $\left(p_{k} / q_{k}\right)_{k \geq-1}$ of this new expansion of $x$, and let $\left(r_{k} / s_{k}\right)_{k \geq-1}$ be the sequence of convergents of the 'old' expansion of $x$, i.e., (8). It was shown in [K91] that the sequence of vectors $\left(p_{k} / q_{k}\right)_{k \geq-1}$ is obtained from $\left(r_{k} / s_{k}\right)_{k \geq-1}$ by removing the term $\left(r_{n} / s_{n}\right)$, i.e.,

$$
\left(\frac{p_{k}}{q_{k}}\right)_{k \geq-1}=\left(\frac{r_{-1}}{s_{-1}}, \frac{r_{0}}{s_{0}}, \ldots, \frac{r_{n-1}}{s_{n-1}}, \frac{r_{n+1}}{s_{n+1}}, \ldots\right) .
$$

2.1.2. Insertions. An insertion is an operation that can be considered as inverse to singularization. An insertion is based upon the identity

$$
a+\frac{1}{b+\xi}=a+1+\frac{-1}{1+\frac{1}{b-1+\xi}},
$$

where $\xi \in[0,1)$ and $a, b$ are positive integers with $b \geq 2$.

Let (8) be a continued fraction expansion of $x \in[0,1)$, with $\left(r_{k} / s_{k}\right)_{k \geq-1}$ as its sequence of convergents, and suppose that for some $n \geq 0$,

$$
a_{n+1}>1, \quad \varepsilon_{n}=1 .
$$


An insertion 'between' $a_{n}$ and $a_{n+1}$ will change (8) into

$$
\left[a_{0} ; \varepsilon_{0} / a_{1}, \ldots, \varepsilon_{n-1} /\left(a_{n}+1\right),-1 / 1,1 /\left(a_{n+1}-1\right), \ldots\right] .
$$

Let $\left(p_{k} / q_{k}\right)_{k \geq-1}$ be the convergents relative to this last expansion. Using some matrix identities it was shown in [K91] that the sequence $\left(p_{k} / q_{k}\right)_{k \geq-1}$ is obtained from $\left(r_{k} / s_{k}\right)_{k \geq-1}$ by inserting the term $\left(\left(r_{n}+r_{n-1}\right) /\left(s_{n}+s_{n-1}\right)\right)$ before the $n$th term, i.e.,

$$
\left(\frac{p_{k}}{q_{k}}\right)_{k \geq-1}=\left(\frac{r_{-1}}{s_{-1}}, \frac{r_{0}}{s_{0}}, \ldots, \frac{r_{n-1}}{s_{n-1}}, \frac{r_{n}+r_{n-1}}{s_{n}+s_{n-1}}, \frac{r_{n}}{s_{n}}, \ldots\right) .
$$

Every time we insert between $a_{n}$ and $a_{n+1}$ we decrease $a_{n+1}$ by 1, i.e. the new $(n+2)$ th digit equals $a_{n+1}-1$. This implies that for every $n$ we can insert between $a_{n}$ and $a_{n+1}$ at most $a_{n+1}-1$ times.

On the other hand, suppose that $a_{n+1}=1$ and that we singularize it. Then both $a_{n}$ and $a_{n+2}$ will be increased by 1 , so we can singularize at most one out of two consecutive digits. For an example, see [HK02], where algorithms based upon singularizations and insertions are given to change the regular expansion of any $x \in[0,1)$ into an odd or an even continued fraction expansion.

In fact, in HK02 two kinds of insertions were introduced. For our purposes, the insertion described by (11) suffices.

2.2. The $D$-map vs. singularizations and insertions. For $n \in \mathbb{N}$, let $x \in I_{n}:=(1 /(n+1), 1 / n]$, so that the RCF-expansion of $x$ looks like

$$
x=\frac{1}{n+\frac{1}{\ddots}},
$$

and suppose that $x \in I_{n} \cap D \neq \emptyset$. Consider the following two cases:

2.2.1. The case $x \in\left(\frac{1}{n+1}, \frac{2}{2 n+1}\right] \cap D$. In this case, due to the definitions of $a_{n}$ and $T$ (cf. (2) and (3)), the RCF-expansion of $x$ is given by

$$
x=\frac{1}{n+\frac{1}{1+\frac{1}{a_{3}+\xi}}}=\left[0 ; n, 1, a_{3}, \ldots\right],
$$

where $\xi \in[0,1)$ and where the first partial quotient $a_{1}$ equals $n$ due to the fact that $x \in(1 /(n+1), 1 / n]$, and the second partial quotient $a_{2}$ equals 1 as $T(x) \in[1 / 2,1]$; see Figure 4 .

Singularizing the second digit, equal to 1 , in the previous expansion we find

$$
x=\frac{1}{n+1+\frac{-1}{a_{3}+1+\xi}}=\left[0 ; 1 /(n+1),-1 /\left(a_{3}+1\right), \ldots\right] .
$$




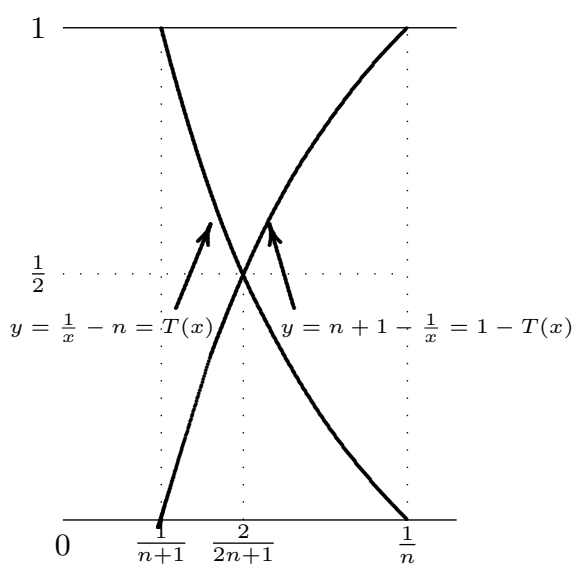

Fig. 4. $D$-map and regular map in the interval $\left(\frac{1}{n+1}, \frac{1}{n}\right]$

Let us now consider the $D$-expansion of $x$. Since $x \in(1 /(n+1), 1 / n] \cap D$, the $D$-expansion of $x$ will look like

$$
x=\frac{1}{n+1+\frac{-1}{\ddots}} .
$$

Let us compute $T_{D}(x)$ to see how to continue. We have

$$
T_{D}(x)=n+1-\frac{1}{x}=1-T(x)=1-\frac{1}{1+\frac{1}{a_{3}+\xi}}=\frac{1}{a_{3}+1+\xi},
$$

so that the $D$-expansion of $x$ is

$$
x=\frac{1}{n+1+\frac{-1}{a_{3}+1+\xi}},
$$

which is equal to $\left[13 ; T_{D}\right.$ acts as a singularization on $\left(\frac{1}{n+1}, \frac{2}{2 n+1}\right] \cap D$.

2.2.2. The case $x \in\left(\frac{2}{2 n+1}, \frac{1}{n}\right] \cap D$. In this case the RCF-expansion of $x$ is given by

$$
x=\frac{1}{n+\frac{1}{a_{2}+\xi}},
$$

where $\xi \in[0,1]$ and $a_{2} \geq 2$ because $T(x) \leq 1 / 2$; see Figure 4 .

An insertion after the first partial quotient yields

$$
x=\frac{1}{n+1+\frac{-1}{1+\frac{1}{a_{2}-1+\xi}} .}
$$


Going to the $D$-expansion, since $x \in(1 /(n+1), 1 / n] \cap D$, the $D$-expansion of $x$ will look like

$$
x=\frac{1}{n+1+\frac{-1}{\ddots}} .
$$

Computing $T_{D}(x)$ we find

$$
T_{D}(x)=1-T(x)=1-\frac{1}{a_{2}+\xi}=\frac{1}{1+\frac{1}{a_{2}-1+\xi}},
$$

so that the $D$-expansion of $x$ is

$$
x=\frac{1}{n+1+\frac{-1}{1+\frac{1}{a_{2}-1+\xi}}},
$$

which is equal to 14 . Thus we see that $T_{D}$ acts as an insertion on $\left(\frac{2}{2 n+1}, \frac{1}{n}\right]$ $\cap D$.

We showed that the $D$-expansion of a real number $x$ can be obtained from its regular expansions via singularization and insertion. Summarizing, we have the following theorem.

THEOREM 1. Let $x$ be a real irrational number with RCF-expansion (1) (so $\varepsilon_{n}=+1$ for all $n \geq 1$ ), and with 'tails' $t_{n}=T^{n}(x)=\left[0 ; 1 / a_{n+1}\right.$, $\left.1 / a_{n+2}, \ldots\right]$. Let $D$ be a measurable subset of $[0,1)$. Then the following algorithm yields the D-expansion of $x$ :

[1] Let $m:=\inf \left\{m \in \mathbb{N} \cup\{\infty\}: t_{m} \in D\right.$ and $\left.\varepsilon_{k}=1\right\}$. In case $m=\infty$, the $R C F$-expansion of $x$ is also the D-expansion of $x$. In case $m \in \mathbb{N}$ :

(i) If $a_{m+2}=1$, singularize the digit $a_{m+2}$ to get

$$
x=\left[a_{0} ; \ldots, 1 /\left(a_{m+1}+1\right),-1 / a_{m+3}, \ldots\right] .
$$

(ii) If $a_{m+2} \neq 1$, insert $-1 / 1$ after $a_{m+1}$ to get

$$
x=\left[a_{0} ; \ldots, 1 / a_{m+1}+1,-1 / 1,1 /\left(a_{m+2}-1\right), \ldots\right] .
$$

[2] Replace the RCF-expansion of $x$ with the continued fraction obtained in [1], and let $t_{n}$ for $n \geq 1$ denote the 'tails' of this new continued fraction expansion of $x$. Repeat the above procedure.

Since the $D$-expansion of $x$ can be obtained from the RCF-expansion of $x$ via suitable singularizations and insertions, we immediately have the following corollary.

Corollary 2. Let $D \subseteq[0,1)$ be a measurable set and let $x \in[0,1)$. Then the D-continued fraction expansion of $x$ is infinite if and only if $x$ is irrational. 
2.3. More about $D$-expansions. Let $x$ be a real number with sequence $\left(r_{n} / s_{n}\right)_{n}$ of RCF-convergents. It is well-known that the $s_{i}$ 's satisfy the recurrence relations

$$
s_{-1}:=0, \quad s_{0}:=1, \quad \text { and } \quad s_{n}=a_{n} s_{n-1}+s_{n-2}, \quad n \geq 1,
$$

and, in particular, they form an increasing sequence. This is essential to the proof of the convergence of the partial quotients $\left(r_{n} / s_{n}\right)_{n}$ to the number $x$, and justifies the notation (1) (see also Chapter 4 in [DK02]).

This property however no longer holds in the $D$-case, since we saw in 12 that inserting in the $n$th position yields a new continued fraction expansion with sequence of convergents $\left(p_{k} / q_{k}\right)_{k}$, for which $q_{n+1}<q_{n}$. Note that we might even have a chain of insertions, thus finding a new sequence of convergents $\left(p_{n} / q_{n}\right)_{n \geq-1}$, satisfying

$$
\left(\frac{p_{k}}{q_{k}}\right)_{k \geq-1}=\frac{r_{-1}}{s_{-1}}, \ldots, \frac{r_{n-1}}{s_{n-1}}, \frac{r_{n}+r_{n-1}}{s_{n}+s_{n-1}}, \ldots, \frac{m r_{n}+r_{n-1}}{m s_{n}+s_{n-1}}, \frac{r_{n}}{s_{n}}, \frac{r_{n+1}}{s_{n+1}}, \ldots
$$

It is still possible though to prove the convergence. In [I89], Shunji Ito studied the map $S:[0,1) \rightarrow[0,1)$ defined by

$$
S(x)= \begin{cases}\frac{x}{1-x}, & 0 \leq x<1 / 2, \\ \frac{1-x}{x}, & 1 / 2 \leq x<1 .\end{cases}
$$

Ito showed that $S$ yields a sequence of convergents $\left(A_{n} / B_{n}\right)_{n \geq-1}$ converging to $x$, which consists of the RCF-convergents of $x$, and of all the mediants of $x$, i.e., the $S$-convergents $A_{n} / B_{n}$ are of the form either $r_{k} / s_{k}$ (for a certain $k$ ), or

$$
\frac{m r_{k-1}+r_{k-2}}{m s_{k-1}+s_{k-2}} \quad \text { with } 0<m<a_{k}
$$

(clearly a mediant does not exist if $a_{k}=1$ ). One can show that the insertion/singularization mechanism described in Theorem 1 implies that the sequence of $D$-convergents of $x$ forms a subsequence of the $S$-convergents of $x$, and therefore we can still conclude that $x=\lim _{n \rightarrow \infty} p_{n} / q_{n}$, which justifies the notation (6).

A proof that $x=\lim _{n \rightarrow \infty} p_{n} / q_{n}$ along the lines of Chapter 4 in [DK02] can also be given. It is essential that

$$
x=\frac{p_{n}+p_{n-1} T_{D}^{n}(x) \varepsilon_{n}}{q_{n}+q_{n-1} T_{D}^{n}(x) \varepsilon_{n}}
$$

that $\operatorname{gcd}\left(p_{n}, q_{n}\right)=1$ and $p_{n-1} q_{n}-p_{n} q_{n-1}=(-1)^{n} \prod_{k=1}^{n-1} \varepsilon_{k}= \pm 1$ for $n \geq 1$, and that

$$
x-\frac{p_{n}}{q_{n}}=\frac{(-1)^{n}\left(\prod_{k=1}^{n} \varepsilon_{k}\right) T_{D}^{n}(x)}{q_{n}\left(q_{n}+q_{n-1} \varepsilon_{n} T_{D}^{n}(x)\right)} .
$$


From these facts it follows that

$$
\left|x-\frac{p_{n}}{q_{n}}\right|=\left|\frac{t_{n}}{q_{n}\left(q_{n}+q_{n-1} \varepsilon_{n} T_{D}^{n}(x)\right)}\right|<\frac{1}{\left|q_{n}\left(q_{n}+q_{n-1} \varepsilon_{n} T_{D}^{n}(x)\right)\right|} .
$$

After a careful analysis of the relation between the $s_{k}$ 's and the $q_{n}$ 's one finds that $x=\lim _{n \rightarrow \infty} p_{n} / q_{n}$.

2.4. Periodic expansions and quadratic irrationals. A number $x$ is called quadratic irrational if it is a root of a polynomial $a x^{2}+b x+c$ with $a, b, c \in \mathbb{Z}, a \neq 0$, and $b^{2}-4 a c$ not a perfect square (i.e., $x$ is an irrational root of a quadratic equation).

The following result is classical; see e.g. [HW79] for a proof.

Theorem 3. A number $x$ is a quadratic irrational number if and only if $x$ has an eventually periodic regular continued fraction expansion.

Similar to the regular case, we say that a $D$-expansion of $x$ is purely periodic of period-length $m$ if the initial block of $m$ partial quotients is repeated throughout the expansion, that is, $a_{k m+1}=a_{1}, \ldots, a_{(k+1) m}=a_{m}$, and $\varepsilon_{k m+1}=\varepsilon_{1}, \ldots, \varepsilon_{(k+1) m}=\varepsilon_{m}$ for every $k \geq 1$. The notation for such a continued fraction is

$$
x=\left[a_{0} ; \varepsilon_{0} / \overline{a_{1}, \varepsilon_{1} / a_{2}, \ldots, \varepsilon_{m-1} / a_{m}, \varepsilon_{m}}\right] .
$$

An (eventually) periodic continued fraction consists of an initial block of length $n \geq 0$ followed by a repeating block of length $m$ and it is written as

$$
x=\left[a_{0} ; \varepsilon_{0} / a_{1}, \varepsilon_{1} / a_{2}, \ldots, \varepsilon_{n-1} / a_{n}, \varepsilon_{n} / \overline{a_{n+1}, \ldots, \varepsilon_{n+m-1} / a_{n+m}, \varepsilon_{n+m}}\right] .
$$

We have the following result, which is a generalization of Theorem 3 .

TheOREM 4. Let $D$ be a measurable subset in the unit interval. Then a number $x$ is a quadratic irrational number if and only if $x$ has an eventually periodic D-expansion.

Proof. Suppose first that $x$ is purely periodic and is given by (18). Due to (16) and the fact that the $D$-expansion of $x$ is purely periodic, we have

$$
x=\frac{p_{m}+p_{m-1} \varepsilon_{m} x}{q_{m}+q_{m-1} \varepsilon_{m} x},
$$

implying that $x$ is a root of the quadratic equation

$$
q_{m-1} \varepsilon_{m} x^{2}+\left(q_{m}-\varepsilon_{m} p_{m-1}\right) x-p_{m}=0 .
$$

Since the $q_{i}$ s are different from 0 it follows that $x$ is quadratic irrational.

Now let $y=\left[0 ; 1 / b_{1}, \varepsilon_{1} / b_{2}, \ldots, \varepsilon_{p-1} / b_{p}, x\right]$ be periodic, with $x$ as in $(18$ purely periodic. Clearly for every integer $b$ the number $b \pm 1 / x$ is again quadratic irrational. The fact that $y$ is quadratic irrational then follows by finite induction. 
Now suppose that $x$ is a quadratic irrational. We must show that $x$ has an eventually periodic $D$-expansion.

In Section 2.2 we saw how every $D$-expansion of $x$ can be derived via insertion and singularization from the regular continued fraction of $x$. Moreover, given a regular periodic continued fraction $y \in[0,1), y=\left[0 ; b_{1}, \ldots, b_{p-1}\right.$, $\left.\overline{b_{p}, \ldots, b_{p+l-1}}\right]$, recall that between $b_{n}$ and $b_{n+1}$ we can insert at most $b_{n+1}-1$ times. In particular, the set of partial quotients of $y$ is finite.

Consider the orbit of the point $x$, which is the (ordered) set

$$
\mathcal{O}=\left\{T(x), T^{2}(x), \ldots, T^{n}(x), \ldots\right\},
$$

obtained by iterations of the Gauss map $T$. Note that in case $x$ is quadratic irrational, it follows from Theorem 3 that $\mathcal{O}$ is finite.

Recall that with every singularization we loose a point in the orbit, while we get a new point every time we insert. However, the maximum number of insertions between two digits is bounded, since we can insert at most $b_{n+1}-1$ times between $b_{n}$ and $b_{n+1}$. Thus, there must exist $\bar{m}, \bar{n}$ with $\bar{m}>\bar{n}$ such that $t_{\bar{m}}=t_{\bar{n}}$. In particular, the tails of the new $D$-expansion will coincide, i.e., $t_{\bar{m}+1}^{-1}=t_{\bar{n}+1}^{-1}$.

3. Metric properties of $D$-expansions. In this section we investigate the metric properties of $D$-expansions when $D$ is a countable collection of disjoint intervals. We are interested in $T_{D}$-invariant ergodic measures that are absolutely continuous with respect to Lebesgue measure. When $D$ is a countable collection of disjoint intervals, the map $T_{D}$ is an example of the family of transformations studied by M. Rychlik Ry83], who showed the existence of a finite number of exact (hence ergodic) measures absolutely continuous with respect to Lebesgue measure. For completeness, we will describe the general setup and result of Rychlik.

Let $X$ be a totally ordered order-complete set. Open intervals constitute a base of a complete topology in $X$, making $X$ into a topological space. If $X$ is separable, then $X$ is homeomorphic to a closed subset of an interval. Let $\mathcal{B}$ be the Borel $\sigma$-algebra on $X$, and $m$ a fixed regular, Borel probability measure on $X$ (in our case $m$ will be the normalized Lebesgue measure restricted to $X)$. Let $U \subset X$ be an open dense subset of $X$ such that $m(U)=1$. Let $S=X \backslash U$, clearly $m(S)=0$.

Let $T: U \rightarrow X$ be a continuous map, and $\beta$ a countable family of closed intervals with disjoint interiors such that $U \subset \cup \beta$. Furthermore, suppose that for any $B \in \beta$ the set $B \cap S$ consists only of endpoints of $B$, and that $T$ restricted to $B \cap U$ admits an extension to a homeomorphism of $B$ with some interval in $X$. Suppose that $T^{\prime}(x) \neq 0$ for $x \in U$, and let $g(x)=1 /\left|T^{\prime}(x)\right|$ for $x \in U,\left.g\right|_{S}=0$. Let $P: L^{1}(X, m) \rightarrow L^{1}(X, m)$ be the Perron-Frobenius 
operator of $T$,

$$
P f(x)=\sum_{y \in T^{-1} x} g(y) f(y) .
$$

In Ry83 it was proved (among many other things) that if $\|g\|_{\infty}<1$ and $\operatorname{Var} g<\infty$, then there exist functions $\varphi_{1}, \ldots, \varphi_{s}$ of bounded variation such that:

(i) $P \varphi_{i}=\varphi_{i}$;

(ii) $\int \varphi_{i} d m=1$;

(iii) there exists a measurable partition $C_{1}, \ldots, C_{s}$ of $X$ with $T C_{i}=C_{i}$ for $i=1, \ldots, s$;

(iv) the dynamical systems $\left(C_{i}, T_{i}, \nu_{i}\right)$, where $T_{i}=\left.T\right|_{C_{i}}$ and $\nu_{i}(B)=$ $\int_{B} \varphi_{i} d m$, are exact, and $\nu_{i}$ is the unique invariant measure for $T_{i}$, absolutely continuous with respect to $\left.m\right|_{C_{i}}$.

Let us return to the map $T_{D}$ with $D$ a countable union of disjoint intervals. Denote by $m$ the normalized Lebesgue measure on $[0,1)$, and let $S=N \cup M$, where $N=\{1 / n: n \in \mathbb{N}\}$ and $M$ the set of endpoints of elements of $D$. Furthermore, let $U=[0,1) \backslash S$. Note that $T_{D}: U \rightarrow[0,1)$ is continuous, and the image of the restriction of $T_{D}$ to each open interval in $U$ is homeomorphic to an interval.

We have $g(x)=1 /\left|T_{D}^{\prime}(x)\right|=x^{2}$ on $U$, hence $\|g\|_{\infty}<1$ and $\operatorname{Var} g<\infty$. Applying Rychlik's result we obtain the following theorem.

TheOrem 5. Suppose D is a countable union of disjoint intervals. Then $T_{D}$ admits at most a finite number of ergodic exact $T_{D}$-invariant measures absolutely continuous with respect to Lebesgue measure.

Throughout the rest of the section, we consider special classes of sets $D$, where the underlying map satisfies the so-called finite image condition; see condition $(\mathrm{F})$ in Theorem 6 below. In this case we can apply a result by Roland Zweimüller [Zw00] to characterize when there is a unique ergodic measure that is absolutely continuous with respect to Lebesgue measure, and to give the support explicitly; see also the related papers [Th80, Th83] by Max Thaler. For completeness we state the result of Zweimüller.

Theorem 6 (Zweimüller). Let $T:[0,1] \rightarrow[0,1]$, and let $B$ be a collection (not necessarily finite) of nonempty pairwise disjoint open subintervals with $m(\bigcup B)=1$ such that $T$ restricted to each element $Z$ of $B$ is continuous and strictly monotone. Suppose $T$ satisfies the following two conditions:

(A) Adler's condition: $T^{\prime \prime} /\left(T^{\prime}\right)^{2}$ is bounded on $\bigcup B$,

(F) Finite image condition: $T B=\{T Z: Z \in B\}$ is finite.

Then there are a finite number of pairwise disjoint open sets $X_{1}, \ldots, X_{n}$ such that $T X_{i}=X_{i}$ (modulo sets of $m$-measure zero) and $\left.T\right|_{X_{i}}$ is conservative 
and ergodic with respect to $m$. Almost all points of $[0,1] \backslash \bigcup_{i} X_{i}$ are eventually mapped into one of these ergodic components and every ergodic component $X_{i}$ can be written as a finite union of open intervals.

Furthermore, each $X_{i}$ supports an absolutely continuous invariant measure $\mu_{i}$ which is unique up to a constant factor.

Finally, for each $i$, the measure $\mu_{i}$ is $\sigma$-finite if and only if $X_{i}$ contains a neighborhood of some indifferent fixed point $x$, i.e. $\left|T^{\prime}(x)\right|=1$.

Note that the above theorem says that the support of each ergodic component is a finite union of open intervals. We apply the above theorem to a few examples.

Example 7 . Suppose $D=\bigcup_{i=0}^{\infty}\left(1 /\left(n_{i}+1\right), 1 / n_{i}\right]$, where $\left(n_{i}\right)_{i \geq 0}$ is a sequence of positive integers. It is clear that in this case $[0,1)$ is the only $T_{D}$ forward invariant set. Hence, $T_{D}$ admits a unique ergodic invariant measure equivalent to Lebesgue measure on $[0,1)$. Furthermore, it is finite if and only if $D$ does not contain 1 , and $\sigma$-finite infinite if $1 \in D$.

EXAMPLE 8. Let $\alpha \in(0,1)$, and suppose $D=\bigcup_{n=1}^{\infty}(1 /(n+1), 1 /(n+\alpha)]$; see Figure 1. As mentioned in Subsection 1.2.1, one gets the folded $\alpha$ expansions, which satisfy the conditions of Zweimüller's theorem. It is easily seen that $T_{D}$ has one ergodic component absolutely continuous with respect to Lebesgue measure, which is finite and with support $[0, \max \{\alpha, 1-\alpha\})$. Note that the invariant measure is known only for $\alpha \in[\sqrt{2}-1,1]$, and that for $\alpha<\sqrt{2}-1$ various remarkable phenomena related to the entropy of these $\alpha$-expansions occur; see [LM08, NaNa08]. In fact, the case $\alpha \in[\sqrt{2}-1,1 / 2]$ can be obtained from the natural extension of the nearest integer continued fraction via singularizations and insertions, in a way similar to the procedure described in [K91]. One could 'stretch' this procedure even a little further for $\alpha$ slightly smaller than $\sqrt{2}-1$, thus obtaining the remarkable 'fractal-like' natural extensions described by Luzzi and Marmi in LM08].

Example 9. Let $\alpha \in(0,1)$ and $D=\bigcup_{n=1}^{\infty}[1 /(n+\alpha), 1 /(n+1))$. Then $T_{D}$ also has one ergodic component absolutely continuous with respect to Lebesgue measure, which is $\sigma$-finite and with support $(\min \{1-\alpha, \alpha\}, 1)$.

Example 10. If $D=(2 / 3,1) \cup \bigcup_{n=2}^{\infty}(1 /(n+1), 2 /(2 n+1)]$, then one gets two ergodic components absolutely continuous with respect to Lebesgue measure. One is finite with support $[0,1 / 2]$ (this continued fraction is in fact the folded nearest integer continued fraction), and the other is $\sigma$-finite with support $(1 / 2,1)$ (this one is in essence Ito's mediant map from [189]); see Figure 5 .

We summarize and generalize the above examples in the following theorem. 


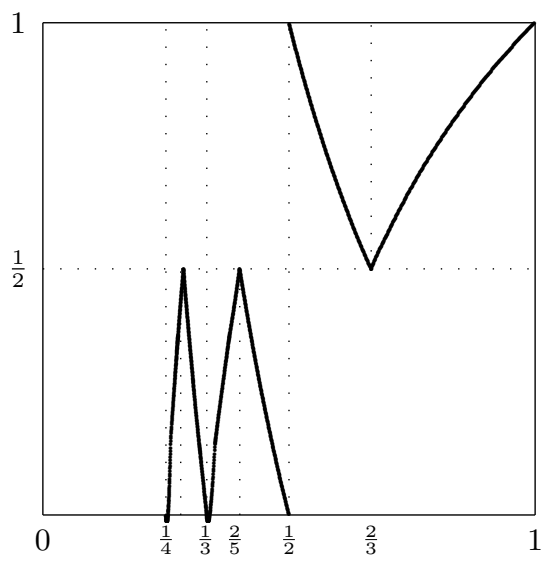

Fig. 5. $\quad D=\left(\frac{2}{3}, 1\right) \cup \bigcup_{n=2}^{\infty}\left(\frac{1}{n+1}, \frac{2}{2 n+1}\right)$

TheOREM 11. Let $D=\bigcup_{n=1}^{\infty} D_{n}$, where for $n \geq 1$ the set $D_{n}$ is either $\left(1 /(n+1), l_{n}\right]$ with $l_{n}<1 / n$, or $\left(l_{n}, 1 / n\right]$ with $l_{n}>1 /(n+1)$, or the whole interval $(1 /(n+1), 1 / n)$, or an empty set. Then the following hold:

(i) If $D$ is as in Example 10 , then $T_{D}$ admits two ergodic components; one with support $[0,1 / 2]$ and with a finite invariant measure, and the other $\sigma$-finite with support $(1 / 2,1)$. This is the only case where one can have two ergodic components.

(ii) If $D_{n}=\left(1 /(n+1), l_{n}\right]$ for $n \geq 1$, with $\left\{T_{D}\left(l_{n}\right): n \in \mathbb{N}\right\}$ finite, then there is a unique ergodic component with support $[0, \alpha]$ for some $0<\alpha<1$.

(iii) If $D_{n}=\left(l_{n}, 1 / n\right]$ for $n \geq 1$, with $\left\{T_{D}\left(l_{n}\right): n \in \mathbb{N}\right\}$ finite, then there is a unique ergodic component with support $[\beta, 1]$ for some $\beta>0$.

(iv) If $D_{1}=[2 / 3,1]$, and if there is at least one $n \geq 2$ for which $D_{n}$ is a proper nonempty subset of $[1 /(n+1), 1 / n]$, then there is a unique ergodic component with support $[1 / 2,1]$.

REMARK 12. 1. If the support of an ergodic measure contains a neighborhood of 1 , then the measure is $\sigma$-finite. Note that $D$ containing a neighborhood of 1 is not enough to have an infinite invariant measure; one needs that the neighborhood of 1 is in the support of the invariant measure. Consider for example the set

$$
D=(q, 1] \cup \bigcup_{n=2}^{\infty}\left(\frac{1}{n+1}, \frac{2}{2 n+1}\right)
$$

with $2 / 3<q<1$; see Figure 6. In this example the map $T_{D}$ maps the interval $[0,1 / 2]$ to itself, and after at most a finite number of steps each point in $(1 / 2,1)$ is mapped to $[0,1 / 2]$. 


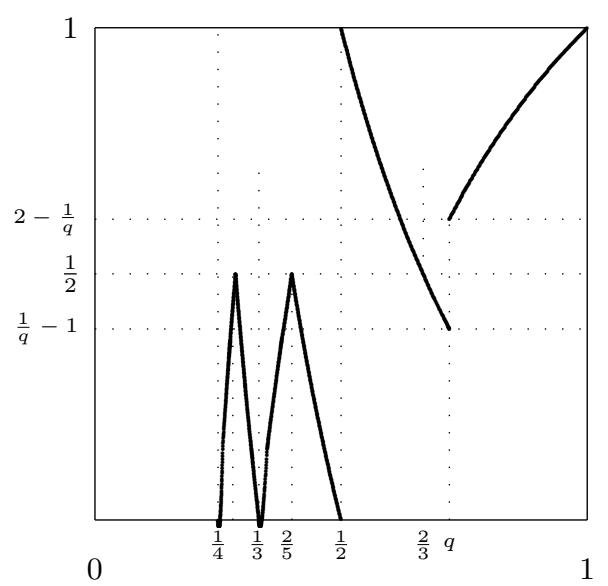

Fig. 6. The case $D=(q, 1] \cup \bigcup_{n=2}^{\infty}\left(\frac{1}{n+1}, \frac{2}{2 n+1}\right)$

2. If $D$ is a combination of cases (ii) and (iii) of Theorem 11, and if $D$ is different from the set discussed in Example 10, then one might have an (erroneous) impression that there is a unique ergodic component with support $[0,1]$. For example, if $D^{c}$ is the complement in $[0,1]$ of the set $D$ from Example 10, then $T_{D^{c}}$ has only one ergodic component, which is the whole unit interval $[0,1]$. However, if $q \in(1 / 2,1)$ is such that $1 / 3<1 / q-1<1 / 2$, and if

$$
D=(q, 1] \cup\left(\frac{2}{5}, \frac{1}{2}\right) \cup \bigcup_{n=3}^{\infty}\left(\frac{1}{n+1}, \frac{2}{2 n+1}\right),
$$

or even $D=(q, 1] \cup(2 / 5,1 / 2)$ (so $D_{n}=\emptyset$ for $n \geq 3$ ), or $D=(q, 1] \cup$ $(2 / 5,1 / 2) \cup[0,1 / 3]\left(\right.$ so $D_{n}=[1 /(n+1), 1 / n]$ for $\left.n \geq 3\right)$, then $T_{D}$ has as support the interval $(1 / q-1,1)$.

4. Computer simulations. To find the density of an invariant measure is in general an extremely hard problem. Most results in this field are merely of an existential nature, i.e. one proves, like we did, that under certain conditions there exists a unique invariant measure, generally assumed equivalent to Lebesgue measure.

In Example 8 we remarked that we are able to obtain the invariant measure of Nakada's $\alpha$-expansions for $\alpha \in[\sqrt{2}-1,1]$ via singularizations and insertions. In particular, in [K91] it is shown that for $\alpha$ between $1 / 2$ and 1 only singularizations suffice, and that the method described in that paper can be applied to many other cases, like the optimal continued fractions (see [BK90]) and Minkowski's diagonal expansions.

Invariant densities are also known for other continued fraction expansions. In [S91], Fritz Schweiger proved that the odd continued fractions pre- 
serve the measure

$$
\mu(A)=\frac{1}{3 \log G}\left(\int_{A} \frac{d x}{G+x-1}+\int_{A} \frac{d x}{G+1-x}\right),
$$

where $G=g^{-1}$, while Rényi [Re57] proved that the measure

$$
\mu(A)=\int_{A} d x / x
$$

is invariant and ergodic for the backward continued fractions map.

In this section we present two methods of estimating the densities of various $D$-expansions. The first method is rather primitive, and counts frequencies based on Birkhoff's ergodic theorem. The second method is accurate and based on a power series method.

4.1. Ergodic method. In general, to get an idea of the density of an invariant measure, we could use Geon Ho Choe's computational approach; see [C0, C05. Using the R program $([\underline{R}])$, we have studied and represented, for particular sets $D$, the distribution of the orbit $\left\{x, T_{D} x, T_{D}^{2} x, \ldots\right\}$ of a random point $x \in[0,1]$ under the map $T_{D}$.

(a)

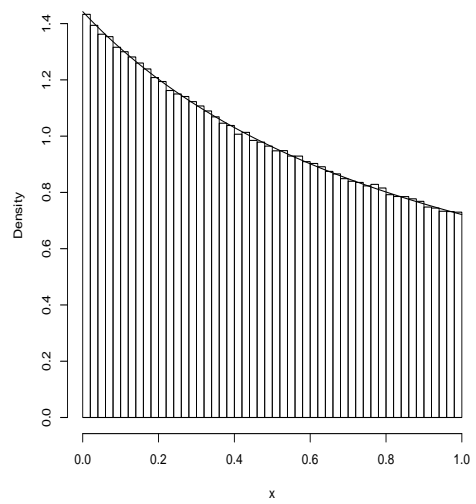

(b)

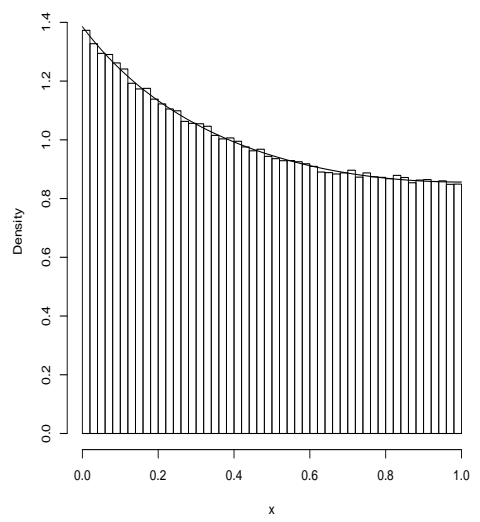

Fig. 7. Invariant measures for (a) the regular continued fraction and (b) the odd continued fraction

Once the ergodicity of the maps is known, the procedure is a straightforward application of Birkhoff's ergodic theorem. In particular we use that, for a measurable set $A$ and sufficiently large $n$,

$$
\mu(A)=\lim _{n \rightarrow \infty} \frac{1}{n} \sum_{i=0}^{n-1} 1_{A}\left(T^{i}(x)\right)
$$


(a) $D=\left[\frac{1}{4}, \frac{1}{3}\right)$

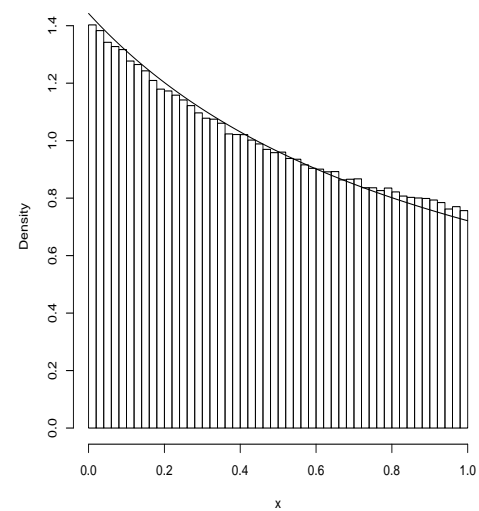

(c) $D=\left[\frac{1}{2}, 1\right)$

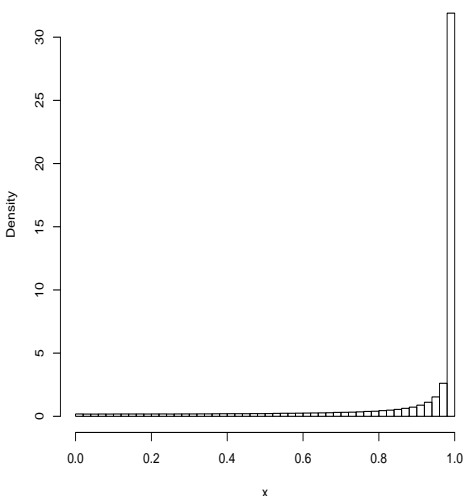

(e) $D=[0.3,0.45)$

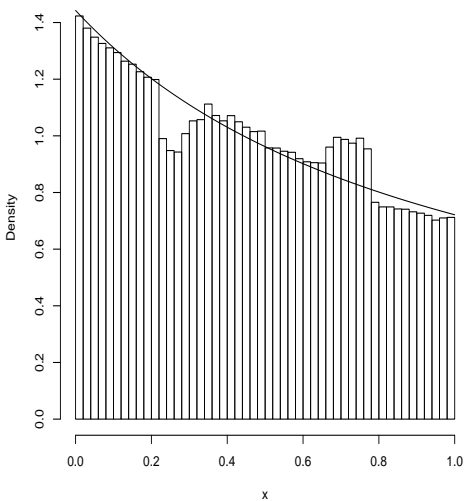

(b) $D=\left[\frac{1}{95}, \frac{1}{94}\right)$

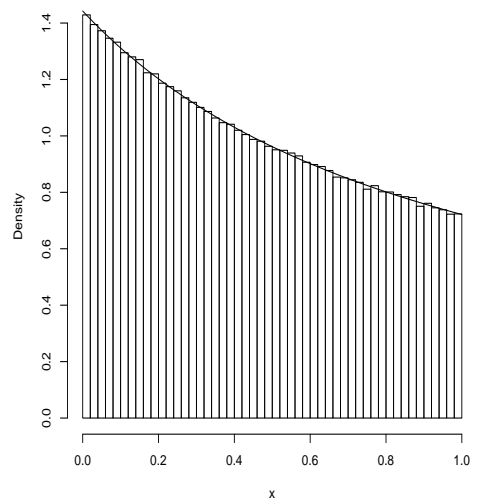

(d) $D=\left[\frac{7}{8}, 1\right)$

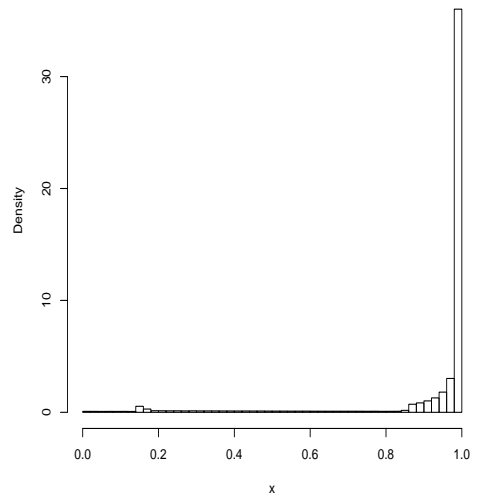

(f) $D=[0.3,0.7)$

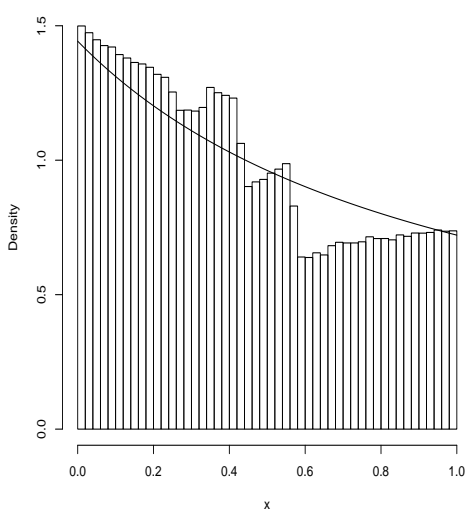

Fig. 8. Some examples of $D$-distributions for particular choices of the set $D$ 
where $1_{A}$ is the characteristic function of $A$; see [C05] for a detailed description of the method used.

Of course, this will only give a rough idea of the invariant densities. However it still seems to be a valuable method. To check the correctness of the code and see how well the algorithm works, we first checked it on some well-known examples: the regular continued fraction expansion and the odd-continued fraction expansions. The results are given in Figure 7 , where the curves drawn in the graphics represent, respectively, the Gauss density (Figure 7(a)) and the Odd-CF density (Figure 7(b)).

All the other results are relative to distributions with unknown densities, and we compare them with the Gauss density. Note, in particular, that Figures $8(\mathrm{c})$ and $8(\mathrm{~d})$ show that, if a neighborhood of 1 is in $D$ and if this neighborhood is also in the forward orbit of the unit interval under $T_{D}$, then 1 is an attractor for the points of the orbit, and the invariant measure appears to be infinite.

Finally, from Figures 8(b), it might seem that the Gauss measure is the invariant measure for the maps $T_{D}$ with $D=[1 / 95,1 / 94)$. It can be very easily checked by direct calculation that this is not correct.

4.2. Power series methods. In this subsection we use a 'power series' method to compute numerically the invariant density and other numerical indicators, including the entropy and the 'Wirsing constant', for a variety of $D$-expansions.

The idea of using power series in computations related to continued fractions has already a considerable history. The bibliography of [B03] amounts to eight pages. Briggs uses this idea to compute, to several hundred decimal places, the Gauss-Kuzmin-Wirsing constant $\lambda=0.3036630029 \ldots$, related to the 'relaxation rate' governing how quickly an initial uniform density 1 for a random variable $X$ settles down to the Gauss density $\frac{1}{\log 2} \frac{1}{1+x}$ in the sequence of probability density functions for $T^{n} X, n \geq 1$.

For folded continued fractions the invariant density is normally not continuous on $[0,1]$, let alone analytic. We cannot use this idea 'straight out of the box'. Nevertheless, it can be adapted to fit our new circumstances. The main idea is to keep track of a number of power series, each representing the density on one of the intervals of continuity of the invariant density, if such intervals exist.

Here, we take a heuristic approach, and focus on a few examples, leaving the general case, and a rigorous justification of the method in its new setting, for another day. [We have that justification for a variation of the approach taken here.] We have reported a handful of digits, truncating further scores of apparently correct digits we obtained. We believe the method is good for about one hundred digits, without need of better hardware or new code. 
After that, most PC's run out of random-access memory. This could be circumvented by a code that swaps data to the hard drive, were there some good reason to get the next hundred digits.

4.2.1. The method and a first example. Our first example is the case $\alpha=$ $1 / 3$ in the examples of $D$-expansions, where $D=\bigcup_{n=1}^{\infty}(1 /(n+1), 1 /(n+\alpha)]$. The map $T_{\alpha}$ is illustrated in Figure 1. Our intervals are $I_{1}=[0,1 / 3], I_{2}=$ $[1 / 3,1 / 2]$, and $I_{3}=[1 / 2,2 / 3]$. For other rational $\alpha$ with $0<\alpha<1$, one would again have a collection of intervals with rational endpoints. If $\alpha$ is quadratic irrational, it may still be possible to use the same approach; we did this with $\alpha=\sqrt{2}-1$. Of course, the endpoints of the intervals will no longer all be rational.

For the method to work, our set of intervals must have the property that each of the inverse branches of $T_{D}$ maps each interval cleanly into exactly one member of the same collection. We can achieve this whenever $D$ is a finite collection of rational intervals that stay away from zero, and also whenever the image under $T$ of $D$ is a finite collection of rational intervals that stay away from zero. We can proceed in much the same spirit as did Briggs and others, but instead of keeping one matrix, we keep several, one for each pair $(J, K)$ of intervals.

Returning to the specific case of $\alpha=1 / 3$, our $T_{\alpha}$, the analogue here of the classical map $T: x \mapsto 1 / x-\lfloor 1 / x\rfloor$, is given by $T(0)=0$, and for values in $(0,2 / 3]$,

$$
T(x)= \begin{cases}1 / x-\lfloor 1 / x\rfloor & \text { if } 1 / x-\lfloor 1 / x\rfloor<1 / 3, \\ \lfloor 1 / x\rfloor+1-1 / x & \text { otherwise. }\end{cases}
$$

The graph of $T$ is a variation of Figure 1 .

The transfer operator for $T$, call it $L$, is given by

$$
L f(x)=\left\{\begin{array}{cc}
\sum_{n=2}^{\infty}(n+x)^{-2} f(1 /(n+x)) & \\
+\sum_{n=2}^{\infty}(n-x)^{-2} f(1 /(n-x)) & \text { if } 0<x \leq 1 / 3, \\
\sum_{n=2}^{\infty}(n-x)^{-2} f(1 /(n-x)) & \text { if } 1 / 3<x \leq 1 / 2, \\
\sum_{n=3}^{\infty}(n-x)^{-2} f(1 /(n-x)) & \text { if } 1 / 2<x \leq 2 / 3 .
\end{array}\right.
$$

The invariant density $g$ for $T$ is the positive eigenfunction of $L$ corresponding to the eigenvalue 1 , normalized by the condition $\int_{0}^{2 / 3} g(x) d x=1$.

Assuming that $g$ is given by three analytic functions, one on each interval, and that these functions have power series expansions about the right endpoints (we will more often use the centers; taking left endpoints fails because zero will be one of the left endpoints and that causes difficulties) of 
the respective intervals, we find that $g$ can be represented as follows:

$$
g(x)= \begin{cases}\sum_{k=0}^{\infty} a_{1 k}(1 / 3-x)^{k} & \text { if } 0<x \leq 1 / 3, \\ \sum_{k=0}^{\infty} a_{2 k}(1 / 2-x)^{k} & \text { if } 1 / 3<x \leq 1 / 2, \\ \sum_{k=0}^{\infty} a_{3 k}(2 / 3-x)^{k} & \text { if } 1 / 2<x \leq 2 / 3 .\end{cases}
$$

We compute the matrix of $L$ with respect to the 'basis' consisting of the union of the following three sets of functions: $B_{1}=\left\{(1 / 3-x)^{k} I_{[0,1 / 3]}(x)\right.$ : $k \geq 0\}, B_{2}=\left\{(1 / 2-x)^{k} I_{[1 / 3,1 / 2]}(x): k \geq 0\right\}$, and $B_{3}=\left\{(2 / 3-x)^{k} I_{[1 / 2,2 / 3]}(x)\right.$ : $k \geq 0\}$. We take $c_{1}=1 / 3, c_{2}=1 / 2$, and $c_{3}=2 / 3$ as our centers of expansion for the series giving $g$ on $I_{1}, I_{2}$, and $I_{3}$, respectively, and we set about computing the matrix whose leading eigenvalue will give the coefficients of $g$ with respect to this 'basis'.

Before getting into detail, we discuss briefly a possible choice of space for $L$ to live on, and some reasons why we should expect a matrix representation. The case of one matrix has been extensively studied in [H06]. The key fact in the classical case is that the maps $1 /(k+x)$ not only carry the interval $[0,1]$ into itself, but they even carry a complex disk that encloses $[0,1]$, say with center 1 and radius 3/2, into itself. Classical complex analysis, based on the fact that contour integrals give coefficients in power series, can then be applied to bound these coefficients. The details in the classical case may be found in $\mathrm{H} 06$.

After some tinkering, weighting coefficients so that the norm assigned to $\left(x-c_{K}\right)^{m}$ depends on $c_{K}$ and $m$ (we have considerable leeway here, and it pays to be cognizant of the fact that $\left(x-c_{K}\right)^{m}$ is uniformly small on $I_{K}$ when $m$ is large), it turns out that in the classical case, the coefficients in our matrix go to zero exponentially with increasing $m$ and $n$. This makes it safe to truncate; the missing coefficients were small anyway. We expect, and experimentally we observe, the same in the case at hand, from the perspective of our norm. That is not to say that the coefficients literally become small - for some choices of $D$, the largest coefficient encountered in the unweighted matrix with $m, n \leq N$ goes to infinity in absolute value as $N$ tends to infinity. This is not that problematic, because our intervals have radius less than $1 / 2$, which helps with convergence.

For $m, n \geq 0$, we let $\zeta(u, v)=\sum_{n=0}^{\infty}(n+v)^{-u}$ be the Hurwitz zeta function with arguments $u, v$.

To continue with the specific case at hand, for $1 \leq J, K \leq 3$, we construct a matrix $A_{J K}$. The matrix $A_{1,2}$, one of nine such matrices, will encode the effect of values of $f$ in the second interval on the values taken by $L f$ in the first interval. We then form a big matrix by quilting together the nine smaller matrices. (If we had just four smaller matrices, $A_{1,1}=[[0,0],[0,0]], A_{1,2}=[[0,0],[0,1]], A_{2,1}=[[1,2],[3,4]]$, and $A_{2,2}=$ 
$[[3,2],[1,0]]$, for instance, our quilted-together matrix $A$ would be $A=$ $[[0,0,0,0],[0,0,0,1],[1,2,3,2],[3,4,1,0]]$.

For the entry at position $(m+1, n+1)$ in $A_{J K}$ we take $A_{J K m n}$ to be the coefficient of $\left(c_{J}-x\right)^{m}$ in Taylor's series expansion about $c_{J}$ of $L_{T}\left(\left(c_{K}-x\right)^{n} I_{\left[c_{K-1}, c_{K}\right]}(x)\right)$. (In effect, indexing begins with 0 rather than 1.)

With this notation, we have

$$
\begin{aligned}
A_{11 m n}= & \sum_{j=0}^{n}\left(\begin{array}{l}
n \\
j
\end{array}\right)\left(-c_{1}\right)^{j}\left(\begin{array}{c}
-2-n+j \\
m
\end{array}\right) \\
& \cdot\left((-1)^{m} \zeta\left(2+n+m-j, 4-c_{1}\right)+\zeta\left(n+m+2-j, 3+c_{1}\right)\right) .
\end{aligned}
$$

As sums of very similar appearance give the other eight matrices, it will save space if we introduce some further notation. Let

We then have

$$
b[m, n, j, K]=\left(\begin{array}{c}
n \\
j
\end{array}\right)\left(-c_{K}\right)^{j}\left(\begin{array}{c}
-2-n+j \\
m
\end{array}\right) .
$$

$$
\begin{aligned}
A_{11 m n}= & \sum_{j=0}^{n} b[m, n, j, 1] \\
& \cdot\left((-1)^{m} \zeta\left(2+n+m-j, 4-c_{1}\right)+\zeta\left(n+m+2-j, 3+c_{1}\right)\right), \\
A_{12 m n}= & \sum_{j=0}^{n} b[m, n, j, 2] \\
& \cdot\left((-1)^{m}\left(3-c_{1}\right)^{-(2+m+n-j)}+\left(2+c_{1}\right)^{-(2+m+n-j)}\right), \\
A_{13 m n}= & (-1)^{m} \sum_{j=0}^{n} b[m, n, j, 3]\left(2-c_{1}\right)^{-(2+m+n-j)} \\
A_{21 m n}= & (-1)^{m} \sum_{j=0}^{n} b[m, n, j, 1] \zeta\left(2+m+n-j, 4-c_{2}\right), \\
A_{22 m n}= & (-1)^{m} \sum_{j=0}^{n} b[m, n, j, 2]\left(3-c_{2}\right)^{-(2+m+n-j)}, \\
A_{23 m n}= & (-1)^{m} \sum_{j=0}^{n} b[m, n, j, 3]\left(2-c_{2}\right)^{-(2+m+n-j)}, \\
A_{31 m n}= & (-1)^{m} \sum_{j=0}^{n} b[m, n, j, 1] \zeta\left(2+m+n-j, 4-c_{3}\right), \\
A_{32 m n}= & (-1)^{m} \sum_{j=0}^{n} b[m, n, j, 2]\left(3-c_{3}\right)^{-(2+m+n-j)} \\
A_{33 m n}= & 0 .
\end{aligned}
$$


On the face of it, then, our $L$ is represented by an ensemble of nine matrices (one of them identically zero). We can shoehorn this situation into the previous context either by interleaving the coefficients, or by simply quilting together our nine square matrices into one. Instead of keeping three lists of coefficients for $g$, we can keep one list. This will not change the eigenvalues of $L_{D}$, nor, if we keep track of things, the underlying function $g$ corresponding to the leading eigenvalue, shown in Figure 9.

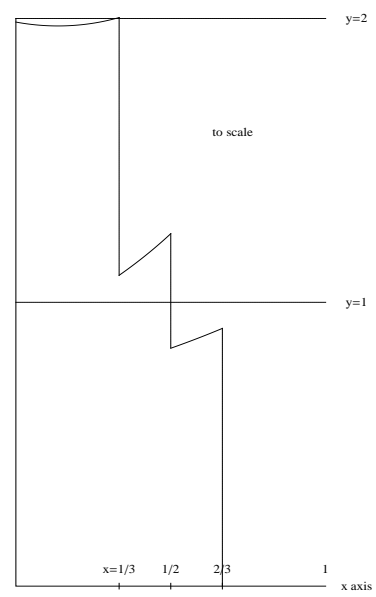

Fig. 9. The underlying function $g$ corresponding to the leading eigenvalue

On the basis of such computations, we expect that $g$ has approximately this series expansion: on $(0,1 / 3)$, with all coefficients positive, the coefficients of $(x-1 / 3)^{k}, 0 \leq k \leq 19$, are

$\begin{array}{lllll}2.00332757 & 0.30681097 & 0.84714782 & 0.34869381 & 0.48266905 \\ 0.28069360 & 0.29529103 & 0.19953556 & 0.18397860 & 0.13403717 \\ 0.11548237 & 0.087616324 & 0.072856661 & 0.056552088 & 0.046169031 \\ 0.036316611 & 0.029376081 & 0.023292992 & 0.018758880 & 0.014948498\end{array}$

We find

$$
\begin{aligned}
& \lim _{x \rightarrow 0^{+}} g(x)=1.987410582312584846, \lim _{x \rightarrow 1 / 3^{-}} g(x)=2.003327565838890855, \\
& \lim _{x \rightarrow 1 / 3^{+}} g(x)=1.09516796670788, \quad \lim _{x \rightarrow 1 / 2^{-}} g(x)=1.24182555817422596, \\
& \lim _{x \rightarrow 1 / 2^{+}} g(x)=0.8381990696715544, \quad \lim _{x \rightarrow 2 / 3^{-}} g(x)=0.90815991310109928 \text {. }
\end{aligned}
$$

The entropy of a dynamical system, such as the one here given by the map $T$ on $[0,2 / 3]$ together with the $T$-invariant measure associated with the 
probability density $g$ on $[0,2 / 3]$, is given by

$$
e=-\int_{x=0}^{2 / 3} g(x) \log \left|T^{\prime}(x)\right| d x .
$$

We find that the entropy is approximately 3.2691593716606420703780299 .

The relaxation rate of the dynamical system is the rate at which arbitrary, reasonably smooth initial pdf's decay to that system's invariant density $g$ under application of $L$. Here, that will be governed by the second eigenvalue of $L$, which is also the leading eigenvalue of the restriction of $L$ to functions $\psi$ for which $\int_{0}^{2 / 3} \psi(x) d x=0$. The second eigenvalue of our approximate matrix for $L$ is

$$
-0.171918835762094311006213249951490933693141706412769
$$

(we expect all places given to be accurate). The associated eigenfunction is shown in Figure 10. Of course, it should have integral zero. This number

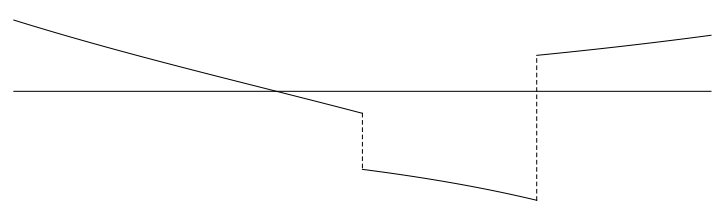

Fig. 10. The associated eigenfunction

is the analogue for our $T$ of the Gauss-Kuzmin-Wirsing constant, whose computation was reported in the reference with which we began our section.

4.2.2. The case $D=[3 / 10,7 / 10]$. Our second example is the case $D=[3 / 10,7 / 10]$. With this choice of $D$, the graph of $T_{D}$ is given in Figure 11, together with its inverse branches: Guided by these graphs and the logic of how the images of intervals fit into each other under the various inverse branches, we dissect $[0,1]$ into ten intervals, with endpoints at $0,1 / 4,3 / 10,1 / 3,3 / 7,1 / 2,4 / 7,2 / 3,7 / 10,3 / 4,1$. We compute 100 matrices, in the same spirit as the nine matrices in the previous case. See Figure 12 for the invariant density for $T_{D}$ (compare this with Figure $8(\mathrm{f})$ ).

We have obtained the following parameters:

$$
\text { entropy }=2.495064971257713861101117578,
$$

Wirsing constant $=0.4504808929430973477827600728336106346$,

$$
\begin{aligned}
\lambda^{\prime}(1) & =-2.4950649712577138611011175784764653641768256, \\
\log \lambda^{\prime \prime}(1) & =3.01514265386003190660939125462523356855698 .
\end{aligned}
$$



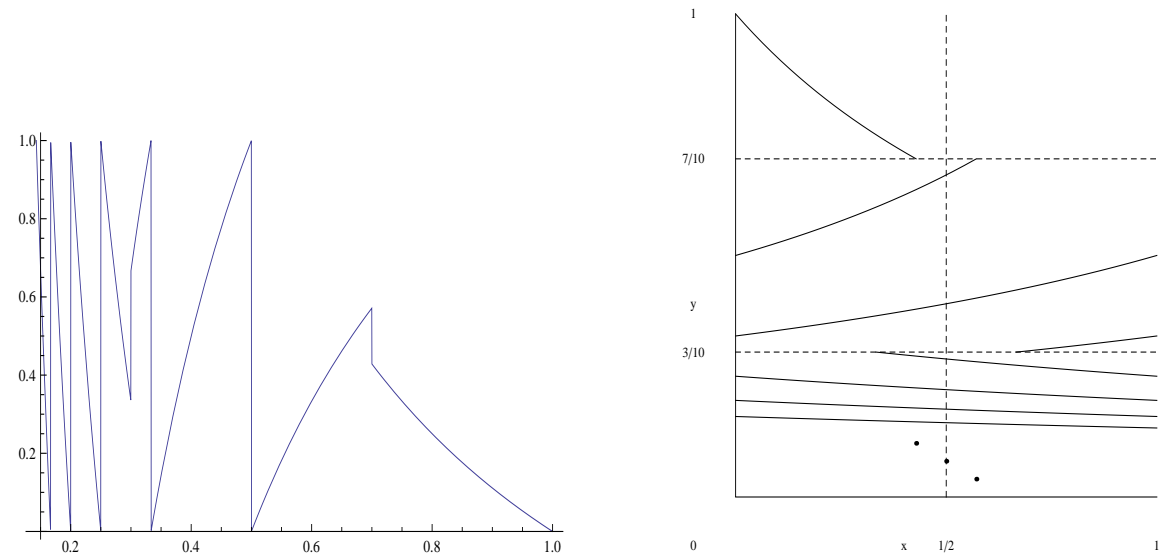

Fig. 11. Left: the graph of $T_{D}$; right: its inverse branches

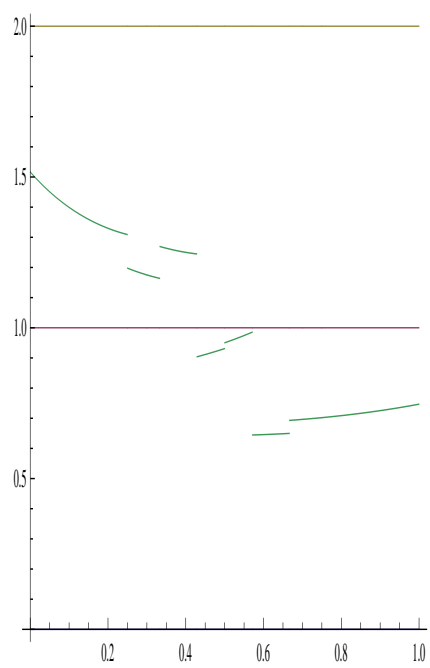

Fig. 12. The invariant density for $T_{D}$ for $D=[3 / 10,7 / 10]$

4.2.3. The case $D=[3 / 10,9 / 20]$. Our third example is $D=[3 / 10,9 / 20]$. We dissect $[0,1]$ into eight intervals by breaking it at $2 / 9,2 / 7,3 / 10,1 / 3$, $1 / 2,2 / 3$, and $7 / 9$. The point of these breaks is that with this dissection, for every positive integer $a$, and every interval $J, 1 /(a+J)$ falls either entirely within the closure of $D$, or within the closure of $D^{c}$, and likewise, for $a \geq 2$, $1 /(a-J)$ falls entirely into one of the above. Also, if $1 /(a+J) \subseteq D^{c}$, then there is a single dissection interval $K$ such that $1 /(a+J) \subset K$, while 
if $1 /(a-J) \subseteq D$, then there is a single dissection interval $K$ such that $1 /(a-J) \subseteq K$.

See Figure 13 for the invariant density for $T_{D}$ (compare with Figure $8(\mathrm{e})$ ). In this case we obtained the following parameters:

$$
\text { entropy }=2.33918451569994744128050557,
$$

Wirsing constant $=0.2927073344680756517102318946443266$, $\log \lambda^{\prime \prime}(1)=3.56660725749380750318932268$.

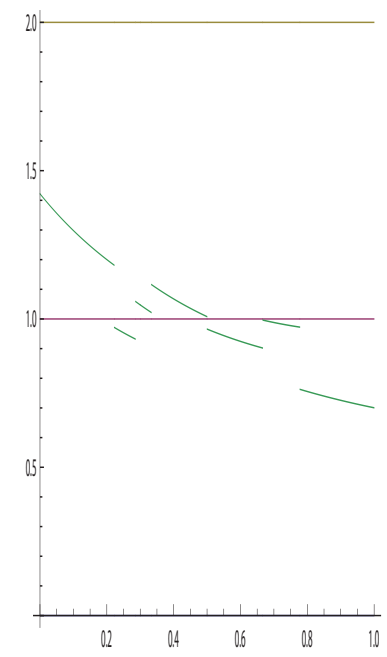

Fig. 13. The invariant density for $T_{D}$ for $D=[3 / 10,9 / 20]$

4.2.4. Nakada-type continued fractions for $\alpha$ near $\sqrt{2}-1$. For our last three examples, we look at choices of $D$ that lead to Nakada-type folded continued fractions associated with a $T_{\alpha}$ with $\alpha$ near $\sqrt{2}-1$. First, we take $D=\bigcup_{n=1}^{\infty}[1 /(n+1), 1 /(n+5 / 12)]$. The invariant density (and with it, the entropy) are already known. The invariant density is shown in Figure 14. The intervals used were demarcated by $0,2 / 7,2 / 5,5 / 12,1 / 2,7 / 12$, but on the numerical evidence, the last two intervals could have been merged. (Our computed values for the invariant density at $1 / 2$, from the left and the right, differed by about $5 \cdot 10^{-103}$ ).

We do have some new numbers: the Wirsing constant for this continued fraction is 0.02269373118279925814257292770843 (no typo!), our computed value for the entropy is

\subsection{7,}

and the second derivative of $\log \lambda(\sigma)$ at $\sigma=1$ is 3.89528129026419411664 . 


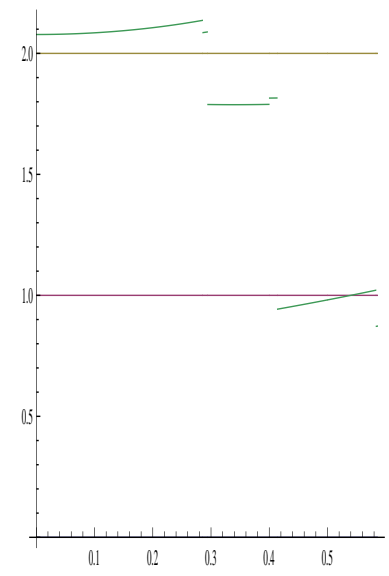

Fig. 14. The invariant density for $T_{\alpha}$ for $\alpha=5 / 12$

For our second example from this batch, we take $\alpha=12 / 29$, so that $D=\bigcup_{n=1}^{\infty}[1 /(n+1), 1 /(n+12 / 29)]$. The invariant density is shown in Figure 15. The Wirsing constant is now 0.1715728752538099 , the entropy is

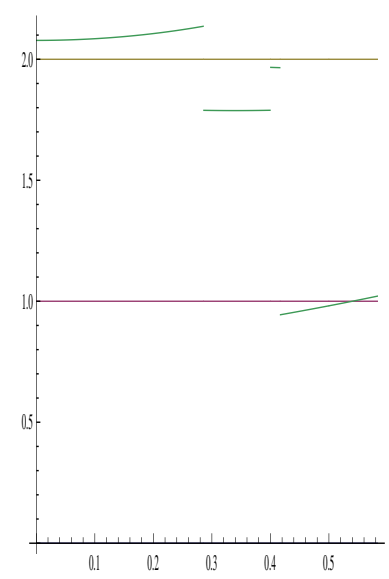

Fig. 15. The invariant density for $T_{\alpha}$ for $\alpha=12 / 29$

3.418315970611, and the counterpart of the final constant in the previous paragraph is now 3.89528129 .

For our final example, we take $\alpha=\sqrt{2}-1$ itself. Our methods give a computed value for the entropy of 
(all digits reported, including those in which we have no confidence), a Wirsing constant of 0.1715728752538099023966 , and for $\log \lambda^{\prime \prime}(1)$ the value 3.895281290264. For $g(0)$, we get 2.0780869212350 . For $g(2-\sqrt{2})$, at the other end, we get 1.022555997219289295539258258 .

Acknowledgments. The third author was sponsored by 'Bezoekersbeurs' 2010/10672/BOO of the Dutch Organization for Scientific Research NWO.

The fourth author was sponsored by a grant of Fondazione Ing. Aldo Gini, Padova.

\section{References}

[AF84] R. L. Adler and L. Flatto, The backward continued fraction map and geodesic flow, Ergodic Theory Dynam. Systems 4 (1984), 487-492.

[B87] W. Bosma, Optimal continued fractions, Indag. Math. 49 (1987), 353-379.

[BK90] W. Bosma and C. Kraaikamp, Metrical theory for optimal continued fractions, J. Number Theory 34 (1990), 251-270.

[B03] K. Briggs, A precise computation of the Gauss-Kuzmin-Wirsing constant, http://keithbriggs.info/documents/wirsing.pdf 2003.

[C00] G. H. Choe, Generalized continued fractions, Appl. Math. Comput. 109 (2000), 287-299.

[C05] -, Computational Ergodic Theory, Algorithms Comput. Math. 13, Springer, Berlin, 2005.

[DK00] K. Dajani and C. Kraaikamp, The mother of all continued fractions, Colloq. Math. 84/85 (2000), 1, 109-123.

[DK02] - - Ergodic Theory of Numbers, Carus Math. Monogr. 29, Math. Assoc. Amer., Washington, DC, 2002.

[H06] D. Hensley, Continued Fractions, World Sci., Hackensack, NJ, 2006.

[HW79] G. H. Hardy and E. M. Wright, An Introduction to the Theory of Numbers, 5th ed., Clarendon Press, New York, 1979.

[HK02] Y. Hartono and C. Kraaikamp, On continued fractions with odd partial quotients, Rev. Roumaine Math. Pures Appl. 47 (2002), 43-62 (2003).

[IK02] M. Iosifescu and C. Kraaikamp, Metrical Theory of Continued Fractions, Math. Appl. 547, Kluwer, Dordrecht, 2002.

[I89] Sh. Ito, Algorithms with mediant convergents and their metrical theory, Osaka J. Math. 26 (1989), 557-578.

[K56] A. Khintchine, Kettenbrüche, Teubner, Leipzig, 1956; see also: A. Khintchine, Continued Fractions, Noordhoff, Groningen, 1963.

[K91] C. Kraaikamp, A new class of continued fraction expansion, Acta Arith. 57 (1991), 1-39.

[LM08] L. Luzzi and S. Marmi, On the entropy of Japanese continued fractions, Discrete Contin. Dynam. Systems 20 (2008), 673-711.

[MMY97] S. Marmi, P. Moussa and J.-C. Yoccoz, The Brjuno functions and their regularity properties, Comm. Math. Phys. 186 (1997), 265-293.

[N81] H. Nakada, Metrical theory for a class of continued fractions transformations, Tokyo J. Math. 4 (1981), 399-426.

[NaNa08] H. Nakada and R. Natsui, The non-monotonicity of the entropy of $\alpha$-continued fraction transformations, Nonlinearity 21 (2008), 1207-1225. 
[NN08] H. Nakada and R. Natsui, The non-monotonicity of the entropy of $\alpha$-continued fraction transformations, ibid. 21 (2008), 1207-1225.

[Pi55] N. Pipping, Semi-regular continued fractions, Nordisk Mat. Tidskr. 3 (1955), 96-106, 127-128.

[P50] O. Perron, Die Lehre von den Kettenbrüchen, Chelsea, New York, 1950.

[R] R Development Core Team, R: A Language and Environment for Statistical Computing, R Foundation for Statistical Computing, Vienna, 2008; http://www.R-project.org

[Re57] A. Rényi, On algorithm for the generation of real numbers, M.T.A. Mat. Fiz. Oszt. Közl. 7 (1957), 265-293 (in Hungarian).

[RS92] A. M. Rockett and P. Szüsz, Continued Fractions, World Sci., River Edge, NJ, 1992.

[Ry83] M. Rychlik, Bounded variation and invariant measures, Studia Math. 76 (1983), 69-80.

[S04] B. Schratzberger, S-expansion in dimension two, J. Théor. Nombres Bordeaux 16 (2004), 705-732.

[S82] F. Schweiger, Continued fractions with odd and even partial quotients, Arbeitber. Math. Inst. Salzburg 1982, no. 4, 59-70.

[S84] - On the approximation by continued fractions with odd and even partial quotients, ibid. 1984, no. 1-2, 105-114.

[S91] - Invariant measures for maps of continued fraction type, J. Number Theory 39 (1991), 162-174.

[Th80] M. Thaler, Estimates of the invariant densities of endomorphisms with indifferent fixed points, Israel J. Math. 37 (1980), 303-314.

[Th83] -, Transformations on [0,1] with infinite invariant measures, ibid. 46 (1983), 67-96.

[Zw00] R. Zweimüller, Ergodic properties of infinite measure-preserving interval maps with indifferent fixed points, Ergodic Theory Dynam. Systems 20 (2000), 1519 1549 .

K. Dajani

Department of Mathematics

Utrecht University

Postbus 80.000

3508 TA Utrecht, the Netherlands

E-mail: k.dajani1@uu.nl

C. Kraaikamp

Delft University of Technology

EWI (DIAM)

Mekelweg 4

2628 CD Delft, the Netherlands

E-mail: c.kraaikamp@tudelft.nl
D. Hensley

Department of Mathematics

Mailstop 3368

Texas A\&M University College Station, TX 77843-3368, U.S.A. E-mail: Doug.Hensley@math.tamu.edu

V. Masarotto

Dipartimento di Matematica Pura ed Applicata Università di Padova 35131 Padova, Italy and

Delft University of Technology

EWI (DIAM)

Mekelweg 4

2628 CD Delft, the Netherlands E-mail: vale.masarotto@libero.it

Received on 23.3.2010

and in revised form on 29.9.2011 
